\title{
Dense Packings of Equal Spheres in a Cube
}

\author{
Th. Gensane \\ Laboratoire de Mathématiques Pures et Appliquées Joseph Liouville, \\ 50, rue F. Buisson, BP 699, 62228 Calais Cedex, FRANCE \\ gensane@lmpa.univ-littoral.fr
}

Submitted: Jun 6, 2003; Accepted: May 20, 2004; Published May 27, 2004

MR Subject Classification: 52C17, 05B40.

\begin{abstract}
We describe an adaptation of the billiard algorithm for finding dense packings of equal spheres inside a domain of the euclidean space. In order to improve the convergence of this stochastic algorithm, we introduce systematic perturbations in it. We apply this perturbed billiard algorithm in the case of $n$ spheres in a cube and display all the optimal and best known packings up to $n=32$. We improve the previous record packings for all $11 \leq n \leq 26$ except $n=13,14,18$. We prove the existence of the displayed packings for $n=11,12,15,17,20,21,22,26,32$, by constructing them explicitly. For example, the graph of the conjectured optimal packing of twenty-two spheres is composed of five octahedrons and four isolated points. We also conjecture that the minimum distance $d_{n}$ between spheres centers of the optimal packings is constant in the range $29 \leq n \leq 32$.
\end{abstract}

\section{Introduction}

We first consider the maximum separation problem in a domain $\mathcal{K}$ of the euclidean space $E^{d}$ : how to spread $n$ points $\mathbf{p}_{1}, \cdots, \mathbf{p}_{n}$ inside $\mathcal{K}$ so that the minimum distance is as large as possible. This formulation is often used by the authors dealing with the disk packing problem which consists in determining the maximum diameter of $n$ equal non-overlapping disks contained in $\mathcal{K}$. A discussion about the equivalence between the two problems can be found in $[\mathrm{M}]$. For other presentations of the subject and related questions, see [CFG, SK, T, Z]. It appears in the mathematical literature that there is no domain where the solution of the problem is obvious. Moreover, it is significant that the regular hexagonal packings of $n=\frac{k(k+1)}{2}$ disks in the equilateral triangle compose the only infinite family of optimal packings known and proved [O]. We note that [GL1] have presented conjectured optimal packings, always in the equilateral triangle, for other infinite classes of $n$.

Recently, some progresses have been made in computer aided proofs [GMPW, N02] as well as in geometric algorithms: the billiard algorithm introduced in [L, LS, GL1, GL2] 
opens a comprehensible and efficient way of finding numerical challenger configurations. Some improvement has also been made in [BDGL]; [GLNO] compare such algorithms with more classical algorithms.

We can think of billiard algorithms as stochastic methods. Indeed, the first version of these algorithms simulates the idealized movement of billiard balls inside a domain, the initial centers of the balls and their directions being randomly fixed. The so obtained packings are the fruit of these probabilistic choices.

In Section 2, we are going to develop the billiard algorithm in a more stochastic way. The hard spheres do not move along straight lines but randomly around themselves, and realize in Algorithm 1 a random walk. In Algorithm 2, the magnitude of the stochastic movements is progressively reduced when the spheres, when growing, yield a rigid position. Simultaneous perturbations of all the spheres will be introduced in Algorithm 3; in Algorithm 4, we alternate those perturbations and Algorithm 2. We finish in Section 3 with some results of sphere packings in a cube and we display all optimal and best known packings for $2 \leq n \leq 32$. We show that contrary to the two-dimensional case, our billiard algorithm and probably the earlier ones are not able to produce all optimal packings with a good accuracy without using perturbations.

In the following, we denote by $\mathcal{K} \subset \mathbb{R}^{d}$ a convex compact subset and $\partial \mathcal{K}$ its boundary, $\mathcal{P}=\left(\mathbf{p}_{1}, \cdots, \mathbf{p}_{n}\right) \in \mathcal{K}^{n}$ a configuration of $n$ points, $d(\mathbf{p}, \mathbf{q})$ the euclidean distance, $B(\mathbf{p}, \varepsilon)=\left\{\underline{\mathbf{q} \in \mathbb{R}^{d}}: d(\mathbf{p}, \mathbf{q})<\varepsilon\right\}$ the open ball of radius $\varepsilon$ centered at $\mathbf{p}$, $S(\mathbf{p}, \varepsilon)=\partial \overline{B(\mathbf{p}, \varepsilon)}$ the sphere of radius $\varepsilon$ centered at $\mathbf{p}$, $f(\mathcal{P})=\min _{i \neq j} d\left(\mathbf{p}_{i}, \mathbf{p}_{j}\right)$ the minimum mutual distance between the points of $\mathcal{P}$, $d_{n}=\max _{\mathcal{P}} f(\mathcal{P})$ the maximum separation distance of $n$ points in $\mathcal{K}$, $\mathcal{K}_{-r}=\{\mathbf{x} \in \mathcal{K}: B(\mathbf{x}, r) \subset \mathcal{K}\}$ the inner parallel body of $\mathcal{K}$, $\mathcal{K}_{r}=\bigcup_{\mathbf{x} \in \mathcal{K}} B(\mathbf{x}, r)$ the outer parallel body (or tubular neighborhood) of $\mathcal{K}$, $\operatorname{pr}_{\mathcal{K}}(\mathbf{p})$ the nearest point in $\mathcal{K}$ to $\mathbf{p} \in \mathbb{R}^{d}$ (the existence of which is guaranteed by the convexity of $\mathcal{K})$, $\mathcal{U}_{\mathcal{K}}$ the uniform law of probability on $\mathcal{K}$.

We recall the following definition found in [D]: to any configuration $\mathcal{P}$, we associate an unoriented simple graph $\mathcal{G}=\mathcal{G}(\mathcal{P})$ whose vertices are the points $\mathbf{p}_{i}$ of $\mathcal{P}$ and whose edges are the segments $\mathbf{p}_{i} \mathbf{p}_{j}$ with $d\left(\mathbf{p}_{i}, \mathbf{p}_{j}\right)=f(\mathcal{P})$. A vertex $\mathbf{p}_{i}$ is called isolated if there is no edge containing $\mathbf{p}_{i}$.

\section{A stochastic algorithm}

We now assume that $\mathcal{K}$ is an erosion-similar body, see [M, p.75]: there is an $r_{0}>0$ such that for all $r \in\left[0, r_{0}\left[\right.\right.$, there exists a composition $S_{r}$ of a rotation, a homothety and a translation which gives $S_{r}(\mathcal{K})=\mathcal{K}_{r}$. It is shown [M, p.78] that under this assumption, the maximum separation problem and the disk packing problem are equivalent. It is therefore 
the same to consider the move of the points $\mathbf{p}_{i}$ inside $\mathcal{K}$ or the move of the hard spheres $S\left(\mathbf{p}_{i}, f(\mathcal{P}) / 2\right)$ of the associated packing in $\mathcal{K}_{f(\mathcal{P}) / 2}$.

Classical billiard algorithms contain statements which compute the directions of the various spheres before and after the shocks. In contrast, our procedures do not include explicit calculations of those directions.

We consider two types of sphere motion.

The first one consists of a random walk of the $n$ spheres. The spheres move one after the other, if a center $\mathbf{p}_{i}$ is moved to $\mathbf{z} \notin \mathcal{K}$ the procedure Random Walking displaces $\mathbf{p}_{i}$ to $p r_{\mathcal{K}}(\mathbf{z})$. If a displaced sphere overlaps another one, the move is not executed. We formalize this through the following algorithm: the variable $N_{a}$ is the number of move attempts, the real number $\varepsilon$ represents the maximal magnitude of the moves, $\alpha$ is the common radius of the $n$ spheres (which is constant during the whole procedure).

\begin{tabular}{|l|}
\hline Algorithm 1: Procedure $\operatorname{Random} \operatorname{Walking}\left(\mathcal{P}, N_{a}, \varepsilon, \alpha\right)$ \\
\hline$\diamond$ For $k$ from 1 to $N_{a}$ do: \\
•Choose randomly (or sequentially) a point $\mathbf{p}_{i}$ in $\mathcal{P}$ \\
- Choose $\mathbf{z}$ following $\mathcal{U}_{B\left(\mathbf{p}_{i}, \varepsilon\right)}$ \\
-If $\mathbf{z} \notin \mathcal{K}$ then $\mathbf{z}:=\operatorname{pr}_{\mathcal{K}}(\mathbf{z})$ \\
-If $\min _{j \neq i} d\left(\mathbf{z}, \mathbf{p}_{j}\right) \geq \alpha$, then $\mathbf{p}_{i}:=\mathbf{z}$ \\
\hline
\end{tabular}

After running the procedure $\operatorname{Random~} \operatorname{Walking}\left(\mathcal{P}, N_{a}, \varepsilon, \alpha\right)$ with $\alpha=f(\mathcal{P})$, we obtain a new configuration $\mathcal{P}$ such that $f(\mathcal{P}) \geq \alpha$; the packing associated to $\mathcal{P}$ is better than the initial packing if the walk allows to improve the smallest distance between the centers $\mathbf{p}_{i}$.

We are now able to give an algorithm similar to the first phase given in [BDGL]: first, the configuration $\mathcal{P}$ is randomly chosen in $\mathcal{K}^{n}$, the real number $\varepsilon_{1}$ is the initial value of $\varepsilon$ in Random Walking, $\varepsilon_{2}$ is the threshold of the procedure.

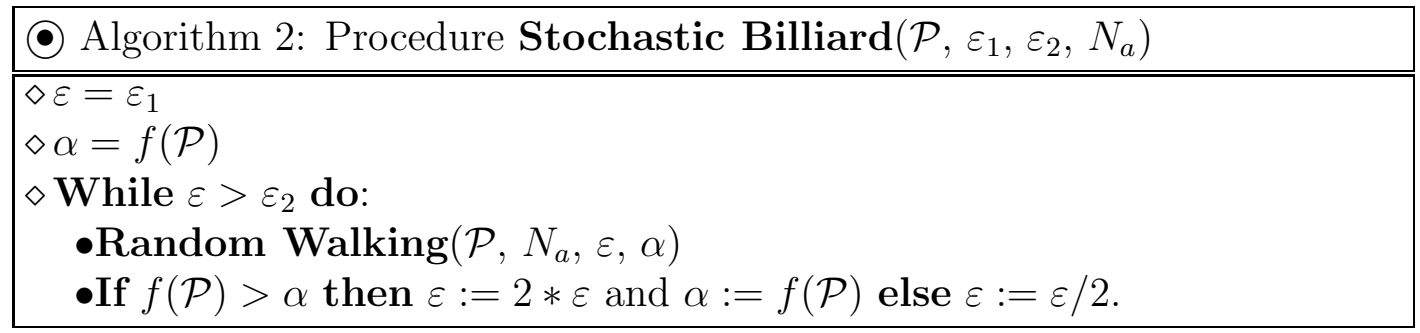

The diameter $f(\mathcal{P})$ of the spheres is increased throughout the procedure Stochastic Billiard as is plainly shown by the last statement $\alpha:=f(\mathcal{P})$ in Algorithm 2.

The second type of motions is a simultaneous perturbation of all the spheres. Those perturbations will be used in a systematic way before the packings become too rigid.

Let us begin with some definitions. As usual, we consider that a configuration $\mathcal{P}$ is locally optimal if

$$
\exists \varepsilon>0, \forall \mathcal{Q} \in \mathcal{K}^{n},\left(\forall i: d\left(\mathbf{q}_{i}, \mathbf{p}_{i}\right)<\varepsilon\right) \Rightarrow f(\mathcal{Q}) \leq f(\mathcal{P}) .
$$

The billiard algorithms (we now include in that class the procedure Stochastic Billiard) lead to configurations which are sometimes locally optimal but in any case solid in 
the following sense: it is impossible to improve the minimum distance of the configuration by moving some spheres of the associated packing, one after the other and without overlaps (a same sphere of center $\mathbf{p}_{i}$ can be moved several times).

Our aim in Algorithm 4 is to produce locally optimal packings, taking into account the degree of freedom of the graph $\mathcal{G}(\mathcal{P})$ as defined in $[\mathrm{D}]$. In fact, we hope that our algorithm will be able to realize a discretization of a path $h$ in the set of solid configurations. More precisely, we can think of $h$ as a continuous map from $[0,1]$ to $\mathcal{K}^{n}$ which satisfies:

- $h(0)=\mathcal{P}$,

- for each $t \in[0,1], h(t)=\left(h_{1}(t), \cdots, h_{n}(t)\right)$ is a solid configuration,

- $f \circ h$ is an increasing map,

- $h(1)$ is a locally optimal configuration.

A solid configuration $\mathcal{P}=\mathcal{P}_{0}$ (or a quasi-solid in the algorithm) will be alternately perturbed by the procedure With Perturbations and improved by the procedure Stochastic Billiard. If the new packing $\mathcal{P}$ is better, we increase the magnitude $\varepsilon$. If an endeavour ends in failure, we restore the previous position $\mathcal{P}_{0}$ and decrease $\varepsilon$. The variable factor gives the threshold $\varepsilon /$ factor (we have used in practice factor $=10^{5}$, it is also possible to fix for instance the ratio $\varepsilon /$ factor to $10^{-12}$ in the call of Stochastic Billiard ). The procedure With Perturbations generates a sequence $\mathcal{P}_{1}, \mathcal{P}_{2}, \cdots, \mathcal{P}_{m}$ of quasi-solid configurations with $f\left(\mathcal{P}_{i+1}\right)>f\left(\mathcal{P}_{i}\right)$. This sequence can be considered as a discretization of a path of solid configurations, although we know that the existence of the continuous map $h$ is far from being proved.

\begin{tabular}{|l|}
\hline Algorithm 3: Procedure $\operatorname{Perturbation}(\mathcal{P}, \varepsilon)$ \\
\hline \hline For $i$ from 1 to $n$ do: \\
- Choose $\mathbf{z}$ following $\mathcal{U}_{B\left(\mathbf{p}_{i}, \varepsilon\right)}$ \\
- If $\mathbf{z} \in \mathcal{K}$ then $\mathbf{p}_{i}:=\mathbf{z}$ else $\mathbf{p}_{i}:=\operatorname{pr}_{\mathcal{K}}(\mathbf{z})$ \\
\hline
\end{tabular}

\begin{tabular}{|l|}
\hline Algorithm $4:$ Procedure With Perturbations $\left(\mathcal{P}, \varepsilon_{1}, \varepsilon_{2}\right.$, factor $)$ \\
\hline \hline$\diamond \varepsilon:=\varepsilon_{1}$ \\
$\diamond \mathcal{P}_{0}:=\mathcal{P}$ \\
$\diamond \alpha_{0}:=f(\mathcal{P})$. \\
$\diamond$ While $\varepsilon>\varepsilon_{2}$ do \\
- Perturbation $(\mathcal{P}, \varepsilon)$ \\
- Stochastic Billiard $\left(\mathcal{P}, \varepsilon, \varepsilon /\right.$ factor, $\left.N_{a}\right)$ \\
- If $f(\mathcal{P})>\alpha_{0}$ then $\alpha_{0}:=f(\mathcal{P})$ and $\varepsilon:=2 * \varepsilon$ \\
else $\mathcal{P}:=\mathcal{P}_{0}$ and $\varepsilon:=\varepsilon / 2$ \\
\end{tabular}

\section{Dense packings of equal spheres in a cube}

The problem of packing $n$ congruent spheres in a unit cube $C$ was raised apparently in 1966 in [S1]. It is shown in that paper that any optimal configuration contains at least one point on every face of $C$ (in fact, the same is true for any parallelotope). The author 
also gave the solutions for $n=2,3,4,5,6,8,9$, the case $n=10$ have been treated in [S2]. These results are the only ones of optimality proved in the cube.

By investigating among the various possible symmetric packings, [Go] described good packings up to twenty-seven spheres. In fact, our computations have shown that all these packings except for $n=13,14,18,27$ are not optimal; we are confident in the optimality of these four surviving cases.

How have we proceeded? It appears that the procedure With Perturbations gives good approximations of local optima. Nevertheless, certain solid configurations calculated by our procedures are both non-locally optimal and tantalizingly rigid (the most difficult computations were for $n=12,25$ and 28). So, we intend to execute the procedure With Perturbations on packings which are not too rigid and close to a global optimum.

In order to find such packings, we have sampled results obtained by the procedure Stochastic Billiard with small values for the parameters $N_{a}$ and $\varepsilon_{2}$ (for instance $N_{a}=$ $600, \varepsilon_{2}=10^{-6}$ and always $\varepsilon_{1}=0.1$ ). The best packing obtained during the sample becomes the starting point of the procedure With Perturbations. In this second phase, and up to thirty-two points, the values $N_{a}=800, \varepsilon_{1}=0.1, \varepsilon_{2}=10^{-12}$ and factor $=10^{5}$ have often given very good results: except for $n=28$, all results in Table 2 have been several times obtained with twelve decimal digits of accuracy.

In the following and relatively to $n$ points, we will denote by $\mathcal{P}^{(n)}$ the best configuration found. For convenience, we will give the coordinates of the points $\mathbf{p}_{i}$ in the cube $[-1,1]^{3}$. But as usual, the values $f\left(\mathcal{P}^{(n)}\right)$ will be given relatively to a unit cube.

We are aware that the earlier billiard algorithms should have found many of the configurations below if they had been simply adapted to the three-dimensional case. Similarly, our algorithm Stochastic Billiard produces, without using systematic perturbations, all optimal and best known disk packings in the square given by [NO1, BDGL]. Nevertheless, the procedure With Perturbations appears to us indispensable in the three-dimensional case for $n=12,19,21,25,28$. The two following examples illustrate that the acceleration of the convergence given by Algorithm 4 is unquestionable.

$\diamond$ For $n=12$, a sample of 10000 runs of Stochastic $\operatorname{Billiard}\left(\mathcal{P}, 0.1,10^{-12}, 2000\right)$ has just given six digits of $f\left(\mathcal{P}^{(12)}\right)$. These calculations have lasted 20 hours on a computer at CPU $800 \mathrm{MHz}$. The algorithm With Perturbations applied to one of the good packings given by the sample gives six new digits in 30 minutes.

$\diamond$ For $n=21,250$ runs of Stochastic Billiard and one hour of calculations are needed to obtain just two digits of $f\left(\mathcal{P}^{(21)}\right)=3(1+\sqrt{3}) / 2$. Then, the algorithm With Perturbations gives in one hour the twelve first digits of this number.

We finish our discussion by considering the cases for which the perturbations are not necessary. We display in Table 1 , for $n \geq 11$, the frequency of obtaining $f\left(\mathcal{P}^{(n)}\right)$ with twelve digits of accuracy, running Stochastic Billiard and the necessary average time to reach them.

Let us finally precise that the association of a small sample of Stochastic Billiard with one run of With Perturbations gives the best found packing of 24 spheres in 4 minutes instead of 40 minutes when we do not use perturbations. 


\begin{tabular}{|c|c|c|c|c|c|c|}
\hline$n$ & 11 & 14 & 15 & 16 & 17 & 18 \\
\hline Frequency & $\frac{1}{140}$ & $\frac{1}{2}$ & $\frac{1}{50}$ & $\frac{1}{40}$ & $\frac{1}{5}$ & $\frac{3}{4}$ \\
\hline Time & $13 \mathrm{~min}$ & $11 \mathrm{~s}$ & $10 \mathrm{~min}$ & $7 \mathrm{~min}$ & $50 \mathrm{~s}$ & $12 \mathrm{~s}$ \\
\hline \hline 20 & 22 & 23 & 24 & 26 & 27 & 32 \\
\hline$\frac{1}{5}$ & $\frac{1}{20}$ & $\frac{1}{25}$ & $\frac{1}{50}$ & $\frac{1}{25}$ & $\frac{1}{10}$ & $\frac{1}{2}$ \\
\hline $1 \mathrm{~min}$ & $5 \mathrm{~min}$ & $3 \mathrm{~min}$ & $40 \mathrm{~min}$ & $12 \mathrm{~min}$ & $5 \mathrm{~min}$ & $1 \mathrm{~min}$ \\
\hline
\end{tabular}

Table 1: Frequency of obtaining $f\left(\mathcal{P}^{(n)}\right)$ with Algorithm 2 and average CPU time.

For each value of $n$, we have tried to give an exact value of the conjectured minimum distance $d_{n}$. For some best packings, the theorem of Pythagoras is sufficient to find an equation verified by $d_{n}$. In that case and for $n=15,17,21,22,32$, the packing is completely described and we know that the packing associated to the displayed graph actually exists.

In any case, the graphs suggested by the computer experiments lead us to conjecture a set of polynomial equations verified by the coordinates of centers $\mathbf{p}_{i}$. Those equations are far from being easily solved, and it is sometimes possible, like in [GMPW], to reduce the order of the system by taking into consideration the symmetries of the configuration and using a Gröbner basis package. For $n=11,12,20,26$, the reduction has been made with Mathematica and we also obtain a proof that the displayed graph corresponds to an existing packing; see below the case $n=11$ for more details. When the reduction fails, we do not dispose of such a proof.

It is clear that our experiments have given configurations of points the coordinates of which are decimal numbers with, say, 12 digits. An exact calculation with 24 digits of accuracy gives the exact value of $x=f\left(\mathcal{P}^{(n)}\right)^{2}$. Now, the graph of the configuration has often just only one edge and we need to conjecture where are the true contacts of the optimal packing close to $\mathcal{P}^{(n)}$. Inspired by [BDGL, p.6], we have adopted the following rule :

$$
\mathbf{p}_{i} \mathbf{p}_{j} \text { is an edge of the displayed graph iff } d^{2}\left(\mathbf{p}_{i}, \mathbf{p}_{j}\right)-x<10^{-12} \text {. }
$$

It is then possible to convince oneself that there does not exist other bonds if, for instance,

$$
d^{2}\left(\mathbf{p}_{i}, \mathbf{p}_{j}\right)-x \notin\left[10^{-12}, 10^{-6}\right] .
$$

For the cases $n=16,19,23,24,25,28$, we have effectively obtained $\left[10^{-12}, 10^{-6}\right]$ with an exact calculation as clear-cut bond identification interval.

We will use ellipsis dots to finish the decimal expansion of any mathematical quantity, as for instance a root of a polynomial. Otherwise, the numbers calculated with a computer will be displayed without ellipsis dots and rounded.

- Cases $n=2$ to $n=6$, see [S1] and Figure 1 .

- Case $n=7$, Figure 1. A configuration $\mathcal{P}$ with $f(\mathcal{P})=1.00091 \cdots$ is proposed in [S1]. But in [S2], Schaer made clear that this result is not optimal and that $d_{7}$ is approximately 1.001089825. We confirm this result and give in Figure 1 the graph of $\mathcal{P}^{(7)}$ in $C$ and its 
associated packing in $C_{f\left(\mathcal{P}^{(7)}\right) / 2}$ which, to our knowledge, have not been published yet. The graph suggests that the configuration is mirror-symmetric. Our experimental result incites us to construct a configuration $\mathcal{P}$ with $\mathbf{p}_{1}=(1,-1,1), \mathbf{p}_{2}=(1-a, 1,1-a)$, $\mathbf{p}_{3}=(-1, a-1,1-a), \mathbf{p}_{4}=(-1,1, b), \mathbf{p}_{5}=(1-a, a-1,-1), \mathbf{p}_{6}=(b, 1,-1)$ and $\mathbf{p}_{7}=$ $(-1,-b,-1)$. The equalities $d^{2}\left(\mathbf{p}_{1}, \mathbf{p}_{5}\right)=d^{2}\left(\mathbf{p}_{5}, \mathbf{p}_{6}\right)=d^{2}\left(\mathbf{p}_{4}, \mathbf{p}_{6}\right)$ give $b=1-a-\sqrt{a^{2}+4 a}$ and $a=-\frac{4}{\sqrt{3}}+\frac{1}{2} \sqrt{\frac{40}{3}+\frac{16}{\sqrt{3}}}$. This explicit configuration has $f(\mathcal{P})=\frac{-4+\sqrt{10+4 \sqrt{3}}}{\sqrt{3}}=$ $1.00108924549 \ldots$, which confirms the result of Schaer and leads us to conjecture that $\mathcal{P}^{(7)}$ is optimal.

- Cases $n=8$ to $n=10$, see [S2] and Figure 2.

- Case $n=11$, Figure 2. Here is the first best packing found having an isolated point; in fact, it can be placed in the nearest corner and then be transformed into a non-isolated point. The configuration admits the same symmetry than $\mathcal{P}^{(7)}$. After observing the graph of $\mathcal{P}^{(11)}$, we define a configuration $\mathcal{P}$ of eleven points having $\mathbf{p}_{1}=(0,0, b-1)$, $\mathbf{p}_{2}=(-1,1,-1), \mathbf{p}_{3}=(-1,1, a-1), \mathbf{p}_{4}=(c, 1,1), \mathbf{p}_{5}=(1, d, e), \mathbf{p}_{6}=(1,-1,1)$. We get a polynomial system of five equations of five unknowns that we have reduced using the Groebner package: $f\left(\mathcal{P}^{(11)}\right)=\frac{a}{2}=0.710116382462 \ldots$ where $a$ is the smallest positive root of the polynomial

$$
\begin{gathered}
58417408-226465792 a+365612032 a^{2}-279190528 a^{3}+208614656 a^{4}- \\
121983488 a^{5}-77183232 a^{6}+107515648 a^{7}-983968 a^{8}-28612736 a^{9}+4587840 a^{10}+ \\
3356352 a^{11}-768432 a^{12}-167776 a^{13}+47376 a^{14}-432 a^{15}+a^{16}
\end{gathered}
$$

The numbers $b, c, d, e$ are expressed in terms of $a$ :

$$
b=\sqrt{a^{2}-2}, c=-1+2 \sqrt{-1+a}, d=f_{1}(a), e=f_{2}(a)
$$

where $f_{1}(a)$ and $f_{2}(a)$ are well-defined but two large to be displayed. Then, with a sufficient accuracy in approximating the root of (1), we obtain bounds for $b, c, d, e$ which show that the points $\mathbf{p}_{i}$ are effectively in the cube. Hence, we conclude that the graph of the constructed configuration $\mathcal{P}$ is actually the displayed graph.

- Cases $n=12$ to $n=14$, Figure 2. Goldberg said in [Go]: "it seems likely that the best arrangements for thirteen spheres and twelve spheres may be obtained by the omission of the appropriate number of spheres from the arrangement $14\{(1,4), 4,(1,4)\}$ ". The last notation means that the best packing of fourteen spheres is composed of three layers, that in the first and last layers there is a sphere surrounded by four equally-spaced spheres, and that four equally-spaced spheres are in the middle layers. Our experiments have confirmed the results for thirteen and fourteen spheres, but have slightly improved the packing of twelve spheres with $f\left(\mathcal{P}^{(12)}\right)=0.707106806467>f\left(\mathcal{P}^{(13)}\right)=f\left(\mathcal{P}^{(14)}\right)=$ $\sqrt{2} / 2=0.707106781186 \ldots$. In fact, Figure 2 shows a deformation of a packing of twelve spheres considered by Goldberg. The best configuration found has two mirror-symmetries. We define a configuration $\mathcal{P}$ of twelve points determined by the points $\mathbf{p}_{1}=(-1, a,-1)$, 
$\mathbf{p}_{2}=(1-b, 1,-1)$ on the first layer, the points $\mathbf{p}_{3}=(-c, 1-d,-c), \mathbf{p}_{4}=(1,0,-e)$ on the middle layer and $\mathbf{p}_{5}=(1,1,1)$ on the upper layer. The seven other points of $\mathcal{P}$ are the symmetric points of $\mathbf{p}_{1}, \cdots, \mathbf{p}_{5}$ with respect to the planes $z=x$ and $y=0$. Here again we have reduced the system of five equations verified by $a, b, c, d, e$ and we have found that $f\left(\mathcal{P}^{(12)}\right)=a=\sqrt{2+2 e+e^{2}} / 2$ where $e$ is the smallest positive root of

$$
\begin{gathered}
20736-289996001280 e+18856111537152 e^{2}-5278350124032 e^{3}-24149235663104 e^{4}- \\
53456543995392 e^{5}+53709113612800 e^{6}-96309967256832 e^{7}+392889686770016 e^{8}- \\
527857748339840 e^{9}+366748643612160 e^{10}-178047190379584 e^{11}+76496366573008 e^{12}- \\
28444213296800 e^{13}+7469161581088 e^{14}-1213853566576 e^{15}+158772078097 e^{16}- \\
43401479624 e^{17}+12816535716 e^{18}-2258516680 e^{19}+277237126 e^{20}- \\
33924440 e^{21}+3309476 e^{22}-154056 e^{23}+2401 e^{24} .
\end{gathered}
$$

Like for $n=11$, we have expressed the real numbers $a, b, c, d$ in terms of $e$ and we conclude that the graph of $\mathcal{P}$ corresponds to an existing packing.

- Case $n=15$, Figure 3. Our computations have given $f\left(\mathcal{P}^{(15)}\right)=0.625=5 / 8$. The configuration is mirror-symmetric, and symmetric with respect to the center $O=\mathbf{p}_{1}$ which is an isolated point. The edges of the graph are obtained from a duplication of the path $((-1,0),(-1 / 4,1),(1,1),(1,-1 / 4),(0,-1))$ on each face of the cube.

- Case $n=16$, Figure 3. The best packing found of sixteen spheres has a three-fold symmetry with respect to a diagonal of the cube. It is clearly obtained by tightening a symmetric packing. We have not succeeded to reduce the system of 8 equations of 8 unknowns given by our graph. Nevertheless, the resolution of this system with Newton's method confirms the coordinates of points.

- Case $n=17$, Figure 3. The first best packing found having a sphere touching twelve other spheres. The configuration is easy to describe and we get $f\left(\mathcal{P}^{(17)}\right)=3 \sqrt{2} / 7$.

- Case $n=18$, Figure 3. We confirm the configuration already given by [Go] in which the optimal configuration of six points in the square appears on three horizontal layers.

- Case $n=19$, Figure 3. The best configuration found of nineteen points is not symmetric and has six isolated points.

- Case $n=20$, Figure 3. The best packing found has six isolated points (two in the heart of the cube), the rigid structure is generated by the central symmetry with respect to 0 , a mirror symmetry and four points $(a,-1,1),(1,-b, 1),(-1,-1, c),(-d,-1,-a)$. The computations lead to $f\left(\mathcal{P}^{(20)}\right)=\sqrt{2}(1-d) / 2$ where $d$ is the smallest positive root of

$$
83-324 x-318 x^{2}+156 x^{3}+3 x^{4}=0 .
$$

For completeness, we give $c=\left(357-683 d+147 d^{2}+3 d^{3}\right) / 304, b=(1-d) / 2, a=1-c-d$ and finally after solving (3), we get

$$
f\left(\mathcal{P}^{(20)}\right)=7 \sqrt{2}+4 \sqrt{6}-2 \sqrt{46+\frac{79}{\sqrt{3}}} .
$$


- Case $n=21$, Figure 4. The best packing found admits a central sphere touching twelve other spheres which are slightly inside the cube. By considering the symmetries of the graph and the three points $\mathbf{p}_{1}=(1,-1,1), \mathbf{p}_{2}=(1-a, 0, b)$ and $\mathbf{p}_{3}=(b, 1-a, 0)$, we obtain $f\left(\mathcal{P}^{(21)}\right)=3 /(2+2 \sqrt{3})$.

- Case $n=22$, Figure 4. The marvelous best configuration of twenty-two points of the figure 4 , is composed of five congruent octahedrons and four isolated points. It is easy to find that $f\left(\mathcal{P}^{(22)}\right)=3 \sqrt{2} / 8$, which is realized for instance by $\mathbf{p}_{1}=(3 / 4,0,0)$ and $\mathbf{p}_{2}=(1,1 / 4,1)$. The packing has three-fold symmetry with respect to any principal diagonal of the cube.

- Case $n=23$, Figure 4. The best configuration found is mirror-symmetric with a central isolated point.

- Case $n=24$, Figure 4. The first best configuration found with eight isolated points.

- Case $n=25$, Figure 4. More and more complexity appears in the sequence of optimal configurations.

- Cases $n=26$ and $n=27$, Figure 4 and 5. The cubic lattice of twenty-seven spheres given by [Go] appears to be optimal in our computations. If we take out the central sphere, there remains a non-solid configuration of twenty-six points: the centers of each face and the middles of each edge can be displaced in order to separate the centers $\mathbf{p}_{i}$. But if all the spheres except the corners are directly displaced towards the center $O$, we just obtain $0.5005 \cdots$ as minimum distance. In fact, our experiments have given 0.501074021252 . Thus, the deformation of the cubic lattice which gives the best packing found of twentysix points is more subtle: the point $(1,0,1)$ is moved by staying inside a face of the cube to $(1-a, 0,1)$, the point $(0,0,1)$ is displaced to $(-c, c, 1-b)$, the displacements of the other points are similar. We find

$$
f\left(\mathcal{P}^{(26)}\right)=\frac{1}{2} \sqrt{1+a^{2}}=0.501074021252 \ldots
$$

where $a$ is the smallest positive root of the polynomial $-16+288 a-728 a^{2}+904 a^{3}-$ $609 a^{4}+208 a^{5}-2 a^{6}-24 a^{7}+7 a^{8}$.

- Cases $n=28$ to $n=32$, Figure 5 and 6 . The coordinates of the points of $\mathcal{P}^{(32)}$ are either \pm 1 or $\pm \frac{1}{3}$. Four spheres inside the cube have twelve spheres touching them. We conjecture after computations that $d_{29}=d_{30}=d_{31}=d_{32}=\sqrt{2} / 3=0.471404520791 \ldots$. Thus, it appears impossible to take out three points from the very regular configuration of thirtytwo points, in order to obtain a non-solid configuration. On the contrary, it is possible to obtain that $d_{28}<d_{32}$ by taking out four points from $\mathcal{P}^{(32)}$ and it seems that our algorithm has given a complex deformation of such a configuration with $f\left(\mathcal{P}^{(28)}\right)=0.471410634842$. 

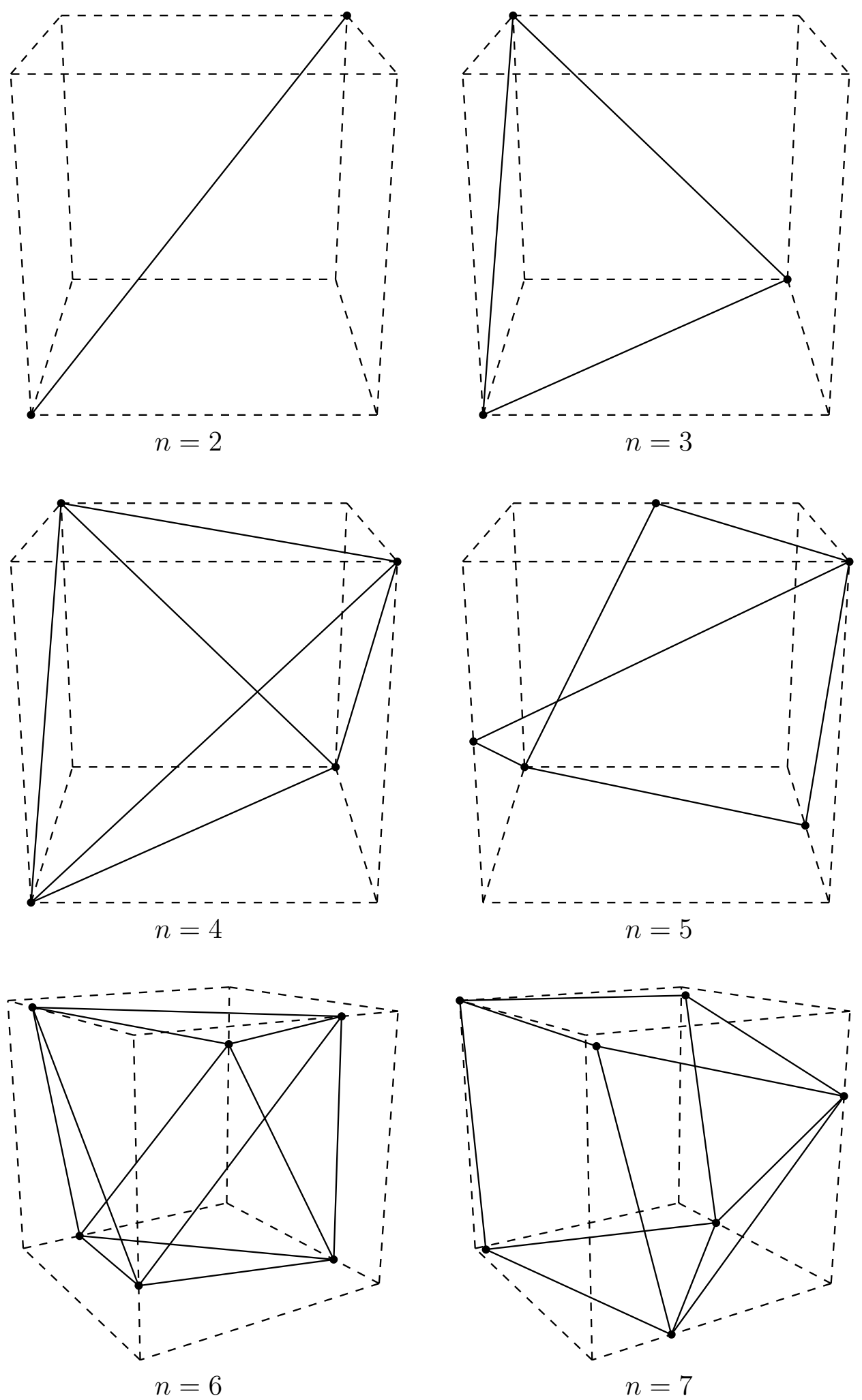

Figure 1: Optimal packings for $2 \leq n \leq 6$ and best known packing for $n=7$. 

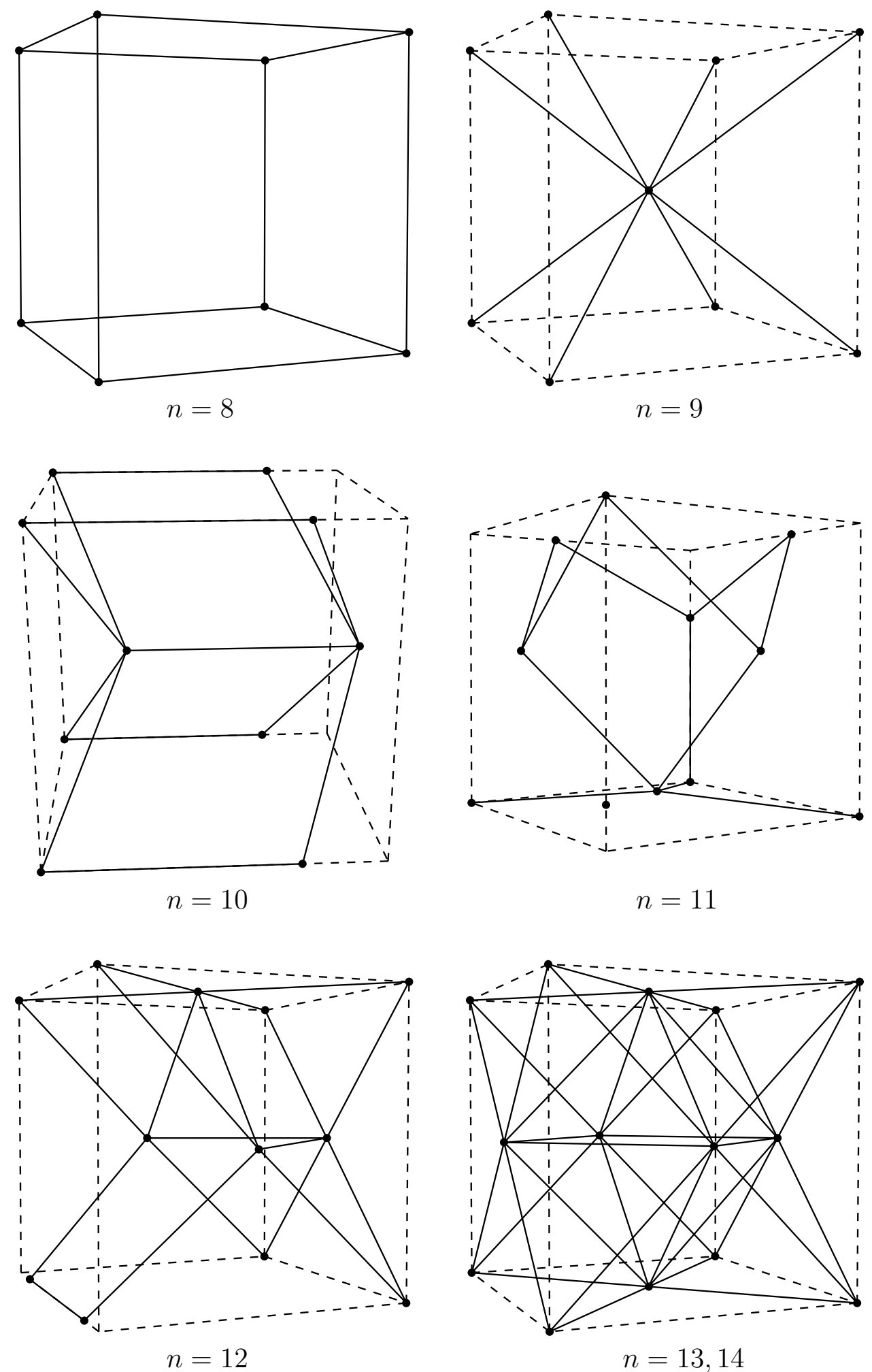

Figure 2: Optimal packings for $8 \leq n \leq 10$ and best known packings for $11 \leq n \leq 14$. 


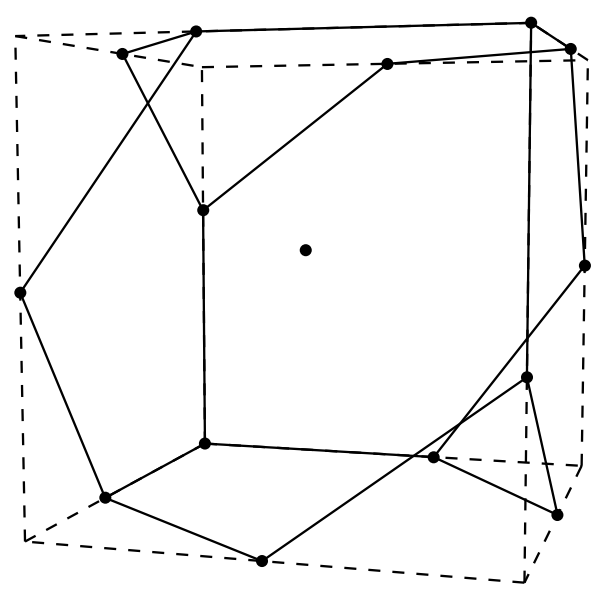

$n=15$

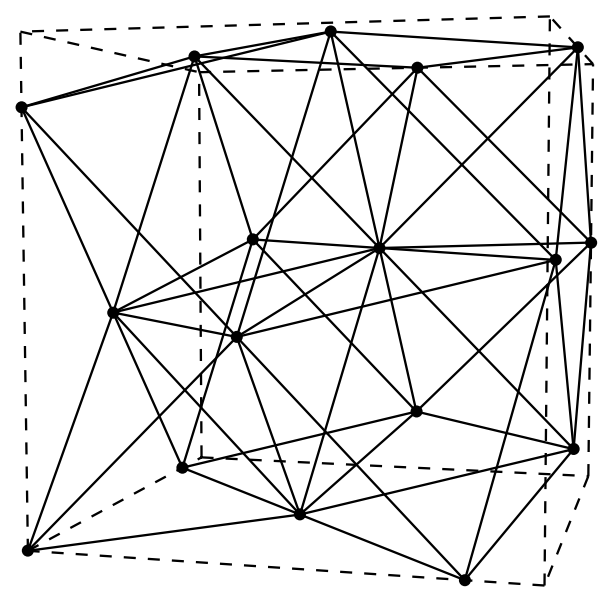

$n=17$

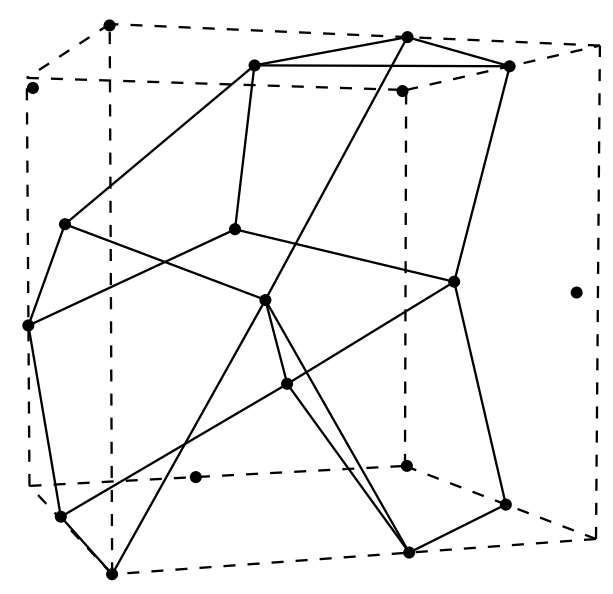

$n=19$

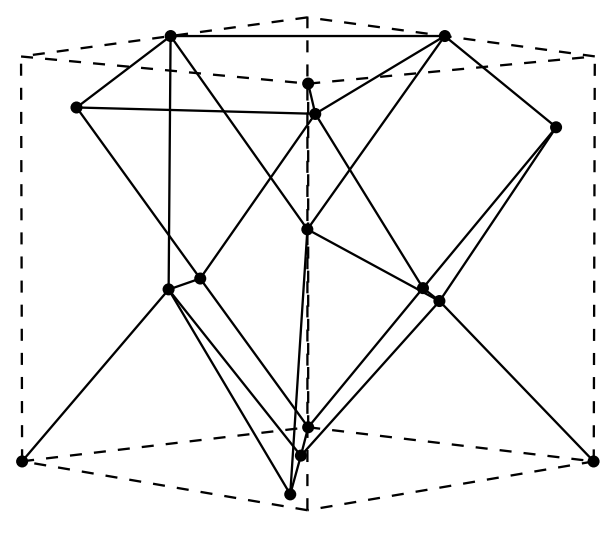

$n=16$

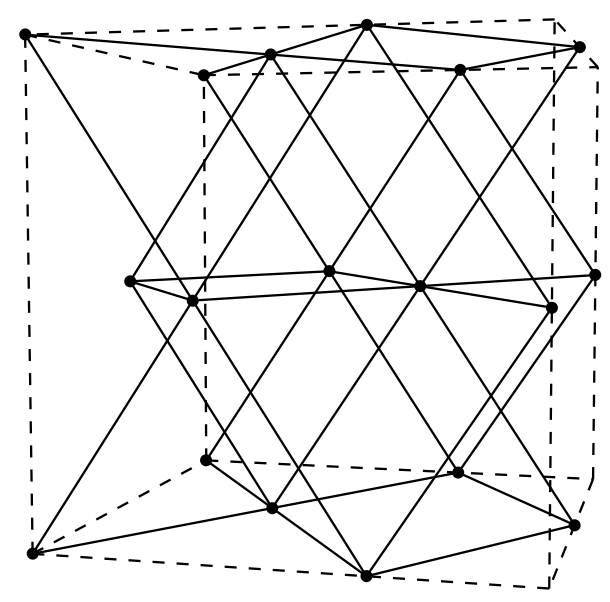

$n=18$

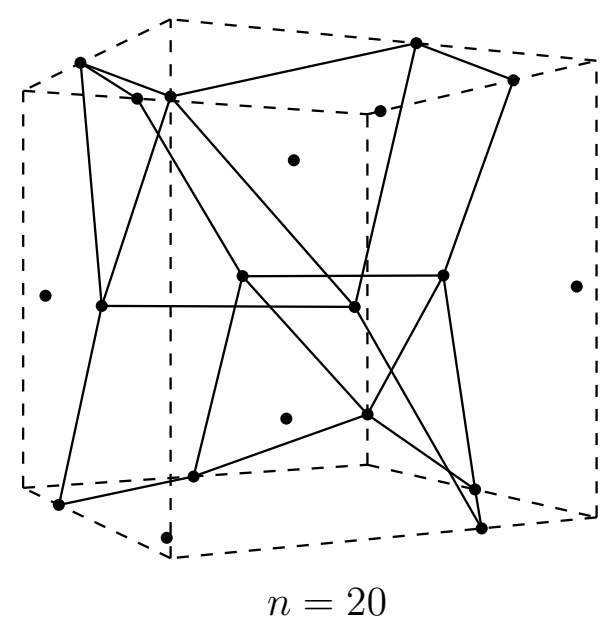

Figure 3: Best known packings for $15 \leq n \leq 20$. 

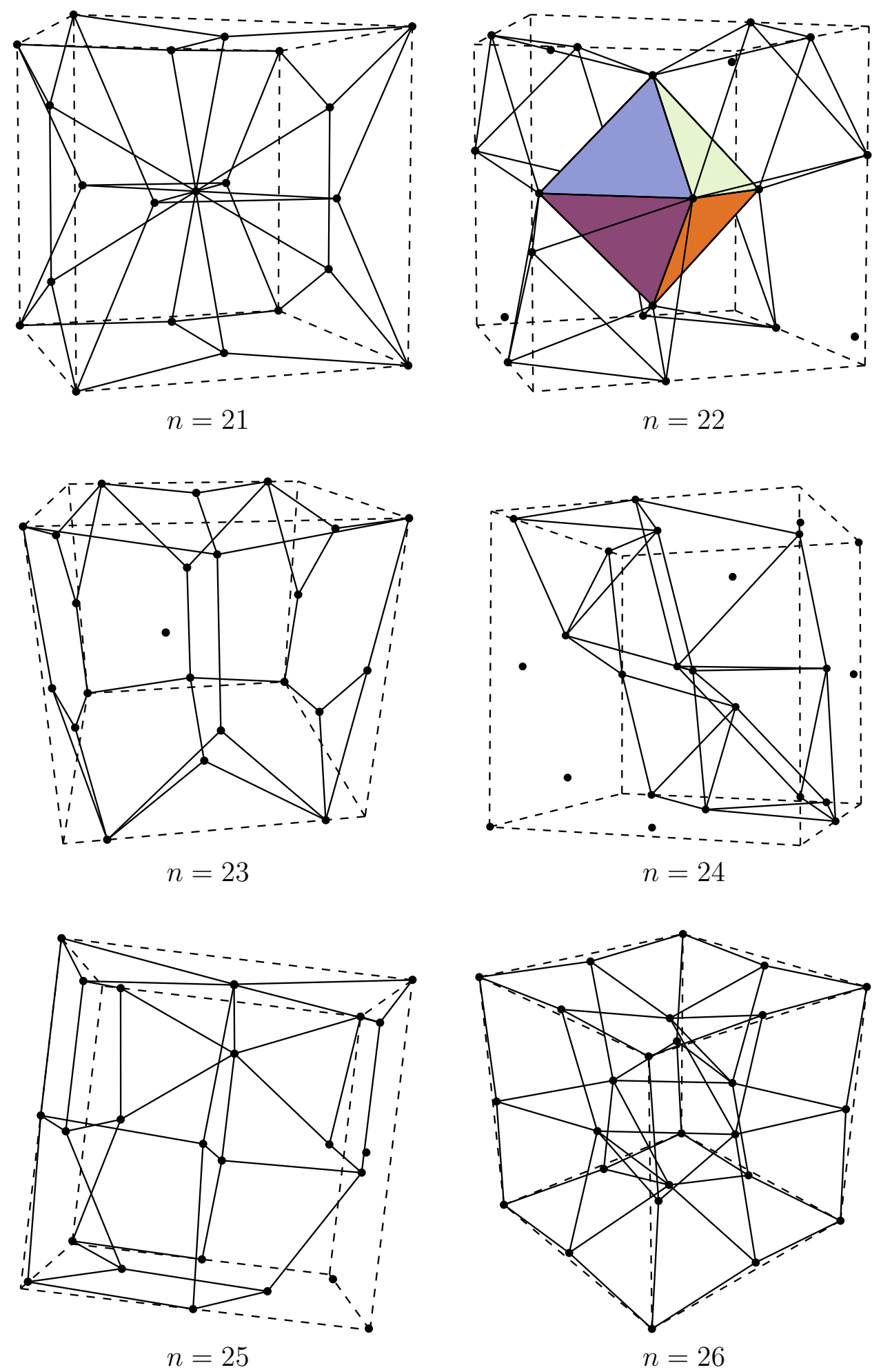

Figure 4: Best known packings for $21 \leq n \leq 26$. 

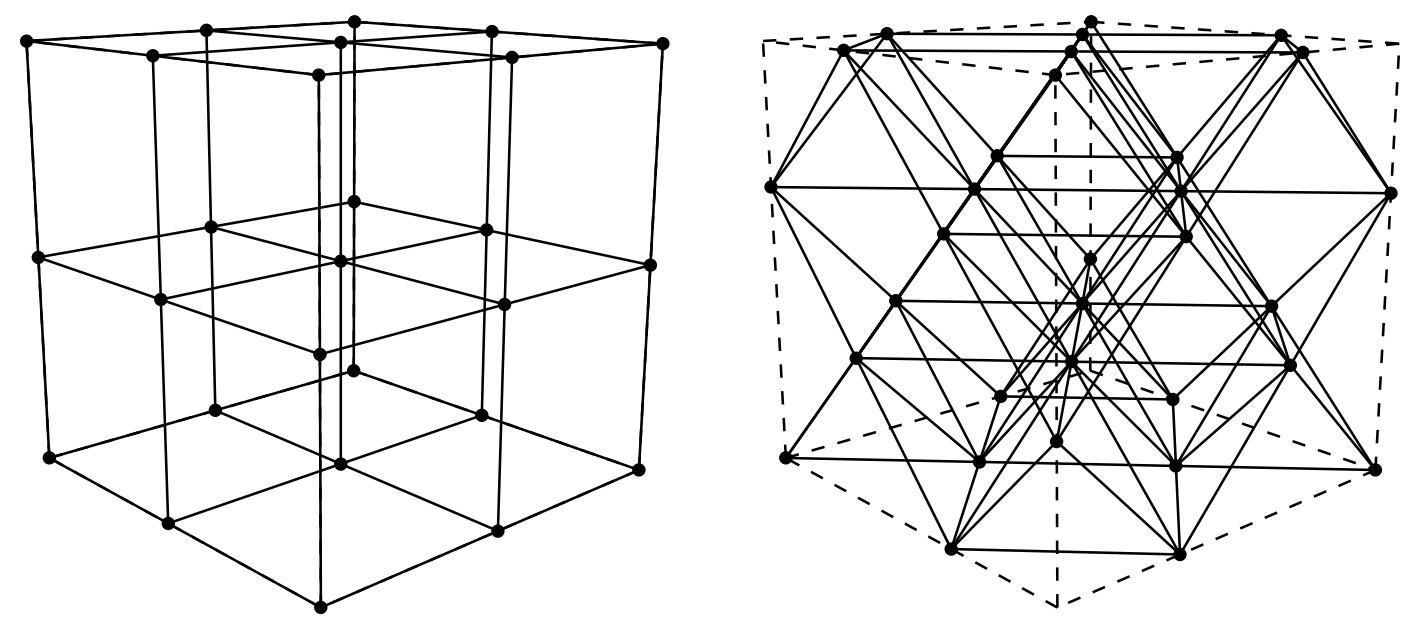

Figure 5: Best known packings for $n=27$ and $29 \leq n \leq 32$.

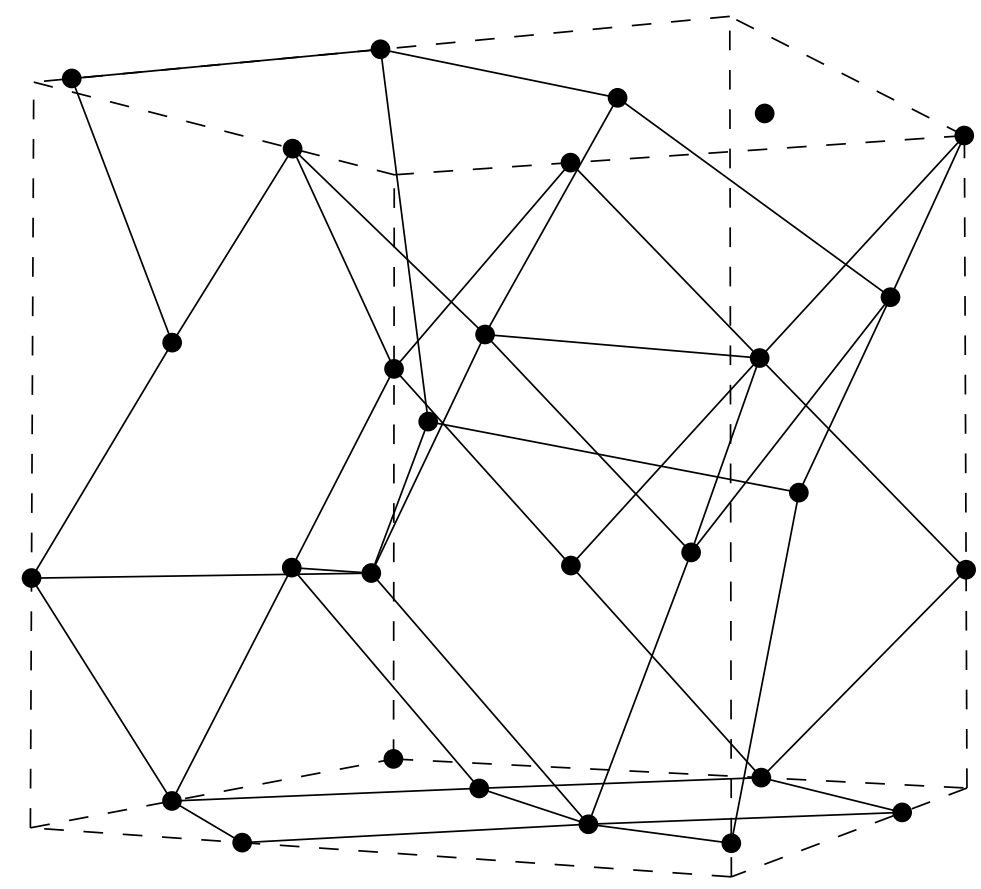

Figure 6: Best known packing for $n=28$ 
We finish this paper with the table of the maximum separation distance $d_{n}$ of $n$ points in a unit cube. By using the same perturbed billiard algorithm in the unit ball $\mathcal{K}=\overline{B(0,1)}$, we have obtained a similar list of results up to $n=40$. We display it in $[\mathrm{G}]$.

\begin{tabular}{|c|c|c|c|c|}
\hline$n$ & $d_{n}$ & Value & Reference & $\#$ of rattlers \\
\hline 1 & 2 & $2.000000000000 \ldots$ & & \\
\hline 2 & $\sqrt{3}$ & $1.732050807568 \ldots$ & & \\
\hline 3 & $\sqrt{2}$ & $1.414213562373 \ldots$ & [S1] & \\
\hline 4 & $\sqrt{2}$ & $1.414213562373 \ldots$ & {$[\mathrm{S} 1]$} & \\
\hline 5 & $\sqrt{5} / 2$ & $1.118033988749 \ldots$ & {$[\mathrm{S} 1]$} & \\
\hline 6 & $3 \sqrt{2} / 4$ & $1.060660171779 \ldots$ & {$[\mathrm{S} 1]$} & \\
\hline 7 & $(-4+\sqrt{10+4 \sqrt{3}}) / \sqrt{3}$ & $1.001089824549 \ldots$ & [S2] & \\
\hline 8 & 1 & $1.000000000000 \ldots$ & {$[\mathrm{S} 1]$} & \\
\hline 9 & $\sqrt{(3) / 2}$ & $0.866025403784 \ldots$ & [S1] & \\
\hline 10 & $3 / 4$ & $0.750000000000 \ldots$ & {$[\mathrm{S} 2]$} & \\
\hline 11 & see $(1)$ & $0.710116382462 \ldots$ & & 1 \\
\hline 12 & see $(2)$ & $0.707106806467 \ldots$ & & \\
\hline 13 & $\sqrt{2} / 2$ & $0.707106781186 \ldots$ & [Go] & \\
\hline 14 & $\sqrt{2} / 2$ & $0.707106781186 \ldots$ & [Go] & \\
\hline 15 & $5 / 8$ & $0.625000000000 \ldots$ & & 1 \\
\hline 16 & & 0.606667120726 & & \\
\hline 17 & $3 \sqrt{2} / 7$ & $0.606091526731 \ldots$ & & \\
\hline 18 & $\sqrt{13} / 6$ & $0.600925212577 \ldots$ & [Go] & \\
\hline 19 & & 0.578209612716 & & 6 \\
\hline 20 & $7 \sqrt{2}+4 \sqrt{6}-2 \sqrt{46+\frac{79}{\sqrt{3}}}$ & $0.554761174904 \ldots$ & & 6 \\
\hline 21 & $3 /(2+2 \sqrt{3})$ & $0.549038105677 \ldots$ & & \\
\hline 22 & $3 \sqrt{2} / 8$ & $0.530330085890 \ldots$ & & 4 \\
\hline 23 & & 0.523539214257 & & 1 \\
\hline 24 & & 0.517638090205 & & 8 \\
\hline 25 & & 0.505135865094 & & 3 \\
\hline 26 & see $(4)$ & $0.501074021252 \ldots$ & & \\
\hline 27 & $1 / 2$ & $0.500000000000 \ldots$ & [Go] & \\
\hline 28 & & 0.471410634842 & & 2 \\
\hline 29 & $\sqrt{2} / 3$ & $0.471404520791 \ldots$ & & \\
\hline 30 & $\sqrt{2} / 3$ & $0.471404520791 \ldots$ & & \\
\hline 31 & $\sqrt{2} / 3$ & $0.471404520791 \ldots$ & & \\
\hline 32 & $\sqrt{2} / 3$ & $0.471404520791 \ldots$ & & \\
\hline
\end{tabular}

Table 2: Maximum separation distance of $n$ points in a unit cube 


\section{References}

[BDGL] D. Boll, J. Donovan, R. L. Graham and B. D. Lubachevsky, Improving dense packings of equal disks in a square, Electron. J. Combin. 7 (2000), \#R46.

[CFG] H. T. Croft, K. J. Falconer and R. K. Guy, Unsolved Problems in Geometry, Springer Verlag, Berlin, 1991.

[D] L. Danzer, Finite point-sets on $S^{2}$ with minimum distance as large as possible, Discrete Math. 60 (1986), 3-66.

[G] Th. Gensane, Dense packings of equal spheres in a larger sphere: a list of result, in Les Cahiers du LMPA J. Liouville 188, June 2003.

[Go] M. Goldberg, On the densest packing of equal spheres in a cube, Math. Mag. 44 (1971), 198-208.

[GL1] R. L. Graham and B. D. Lubachevsky, Dense packings of equal disks in an equilateral triangle: from 22 to 34 and beyond, Electron. J. Combin. 2 (1995), \#A1.

[GL2] R. L. Graham and B. D. Lubachevsky, Repeated patterns of dense packings of equal disks in a square, Electron. J. Combin. 3 (1996), \#R16.

[GLNO] R. L. Graham, B. D. Lubachevsky, K. J. Nurmela, and P.R. J. Östergård, Dense packings of congruent circles in a circle, Discrete Math. 181 (1998), 139-154.

[GMPW] C. de Groot, M. Monagan, R. Peikert, and D. Wurtz, Packing circles in a square: a review and new results, in System Modeling and Optimization (Proc. 15th IFIP Conf. Zurich 1991), Lecture Notes in Control and Information Science, Springer-Verlag, Berlin 180, 45-54, 1992.

[L] B. D. Lubachevsky, How to simulate billiards and similar systems, J. Comput. Phys. 94 (1991), 255-283.

[LS] B. D. Lubachevsky and F. H. Stillinger, Geometric properties of random disk packings, J. Statist. Phys. 60 (1990), 561-583.

[M] H. Melissen, Packing and Covering with Circles, Ph.D. thesis, University of Utrecht, 1997.

[NO1] K. J. Nurmela and P. R. J. Östergård, Packing up to 50 equal circles in a square, Discrete Comput. Geom. 18 (1997), 111-120.

[N02] K. J. Nurmela, P. R. J. Östergård, More optimal packings of equal circles in a square, Discrete Comput. Geom. 22 (1999), 439-457. 
[O] N. Oler, A finite packing problem, Canad. Math. Bull. 4 (1961), 153-155.

[S1] J. Schaer, On the densest packing of spheres in a cube, Canad. Math. Bull. 9 (1966), 265-270, 271-274, 275-280.

[S2] J. Schaer, The densest packing of ten congruent spheres in a cube, in Intuitive geometry (Szeged, 1991), Colloq. Math. Soc. János Bolyai 63 (1994), 403-424. K. Böröczky and G. Fejes Tóth (Eds.), North-Holland, Amsterdam.

[SK] E.B. Saff, A.B.J. Kuijlaars, Distributing many points on a sphere, Math. Intelligencer 10 (1997), 5-11.

[T] T. Tarnai, Spherical circle-packing in nature, practice and theory, Struct. Topology 9 (1984), 39-58.

[Z] Y.M. Zhou, Arrangements of Points on the Sphere, Ph.D. thesis, University of South Florida, 1995. 